\title{
Machine Learning for Challenging EELS and EDS Spectral Decomposition
}

\author{
Thomas Blum ${ }^{1,2, *}$, Jeffery Graves ${ }^{3}$, Michael Zachman², Ramakrishnan Kannan ${ }^{3}$, Xiaoqing Pan ${ }^{1}$, \\ Miaofang $\mathrm{Chi}^{2, *}$ \\ 1. Physics \& Astronomy, University of California, Irvine, Irvine, CA, USA \\ 2. Center for Nanophase Materials Sciences, Oak Ridge National Laboratory, Oak Ridge, TN, USA \\ 3. Computational Data Analytics, Oak Ridge National Laboratory, Oak Ridge, TN, USA \\ * Corresponding Author: tblum@uci.edu, chim@ornl.gov
}

Scanning transmission electron microscopy (STEM) is one of the primary methods of characterizing heterogenous catalysts due to its unprecedented spatial resolution and the ability to perform imaging and chemical analysis simultaneously at the atomic scale. For the characterization of heterogenous catalysts that are composed of metal and oxide support, the atomic configurations are often probed by Z-contrast imaging while the chemical distributions can be revealed by using either electron energy loss spectroscopy (EELS) or energy dispersive X-ray spectroscopy (EDS). However, challenges in characterizing catalysts using STEM lay in detecting the subtle chemical and electronic structure changes on the metal nanoparticle surfaces. Possible overlapping of peaks/edges in EELS and EDS further complicate the analysis, making traditional analysis difficult. Machine learning offers exciting tools for analyzing EELS and EDS spectrum images where traditional analysis techniques struggle. We propose a new procedure for improving the convergence of the non-negative matrix factorization (NMF) dimension reduction algorithm to a meaningful and interpretable result.

As a result of recent developments in machine learning algorithms and increased collaborations between disciplines, there has been an increase in the use of statistical machine learning applications for the study of materials. Among the most popular is principle component analysis (PCA), which produces abstracted representations of spectra because of the orthogonality requirement of the algorithm. This makes the resulting components difficult to interpret. NMF on the other hand, has been shown to produce interpretable results due to its non-negativity constraint on the components and abundance maps. A weakness of conventional NMF, however, is its sensitivity to local minima during convergence. One way to combat this effect is to impose additional constraints, or costs, on the minimization algorithm to reduce the likelihood of settling at a local minimum. Another approach is to use an improved initial guess, which increases the likelihood of the algorithm reaching the global minimum[1].

Here, we combine these techniques to improve the quality of results by using the non-linear "perfect pixel" algorithm, ATGP [2], to generate initial guesses for the joint-non-negative matrix factorization [3] algorithm that augments the cost function of NMF and encourages sparsity in the pixels and smooth transitions. Figures 1 and 2 show that Joint-NMF identified the palladium nanocube in the second component and differentiated two carbon components. The first component is the uniform lacey carbon support and the second component builds in thickness a feature characteristic of carbon contamination. The number of components was estimated by selecting the number of components required to capture the most variance as determined with PCA. This work shows that this new machine learning based algorithm is able to detect overlapping components in EELS spectrum image datasets that are challenging for conventional microscopy data analysis methods. [4] 


\section{References:}

[1] J Graves et al., Submitted (2018)

[2] H Ren, C Chang, IEEE Transactions on Aerospace and Electronic Systems 39 (2004), p. 1232

[3] R Kannan et al., Advanced Structural and Chemical Imaging 4 (2018), p. 6

[4] This work is supported by ORNL's Laboratory Directed Research and Development (LDRD) funds. The microscopy work was performed at ORNL's Center for Nanophase Material Sciences, which is a U. S. Department of Energy Office of Science User Facility.
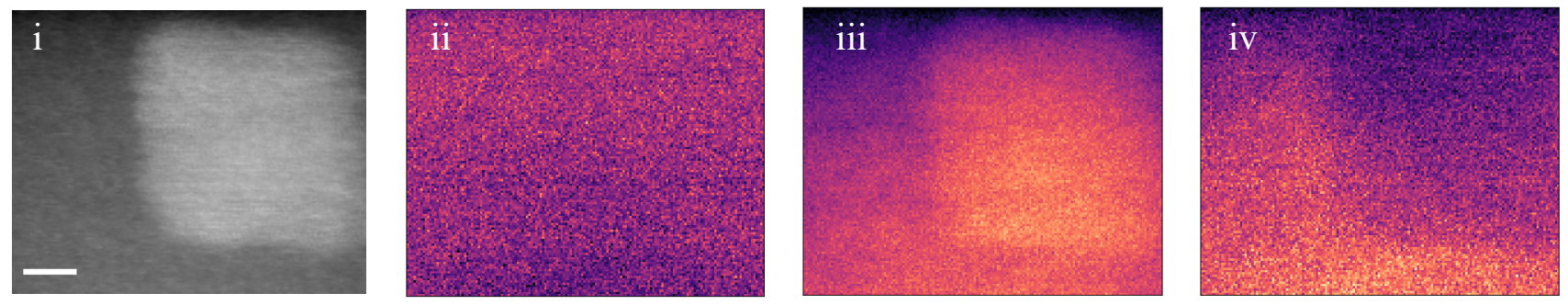

Figure 1. Z-contrast STEM image and component maps of palladium on carbon from Joint-NMF. From left to right: High angle annular darkfield (HAADF)-STEM signal, lacey carbon support, palladium component, carbon contamination. The length of the scale bar is $2 \mathrm{~nm}$.

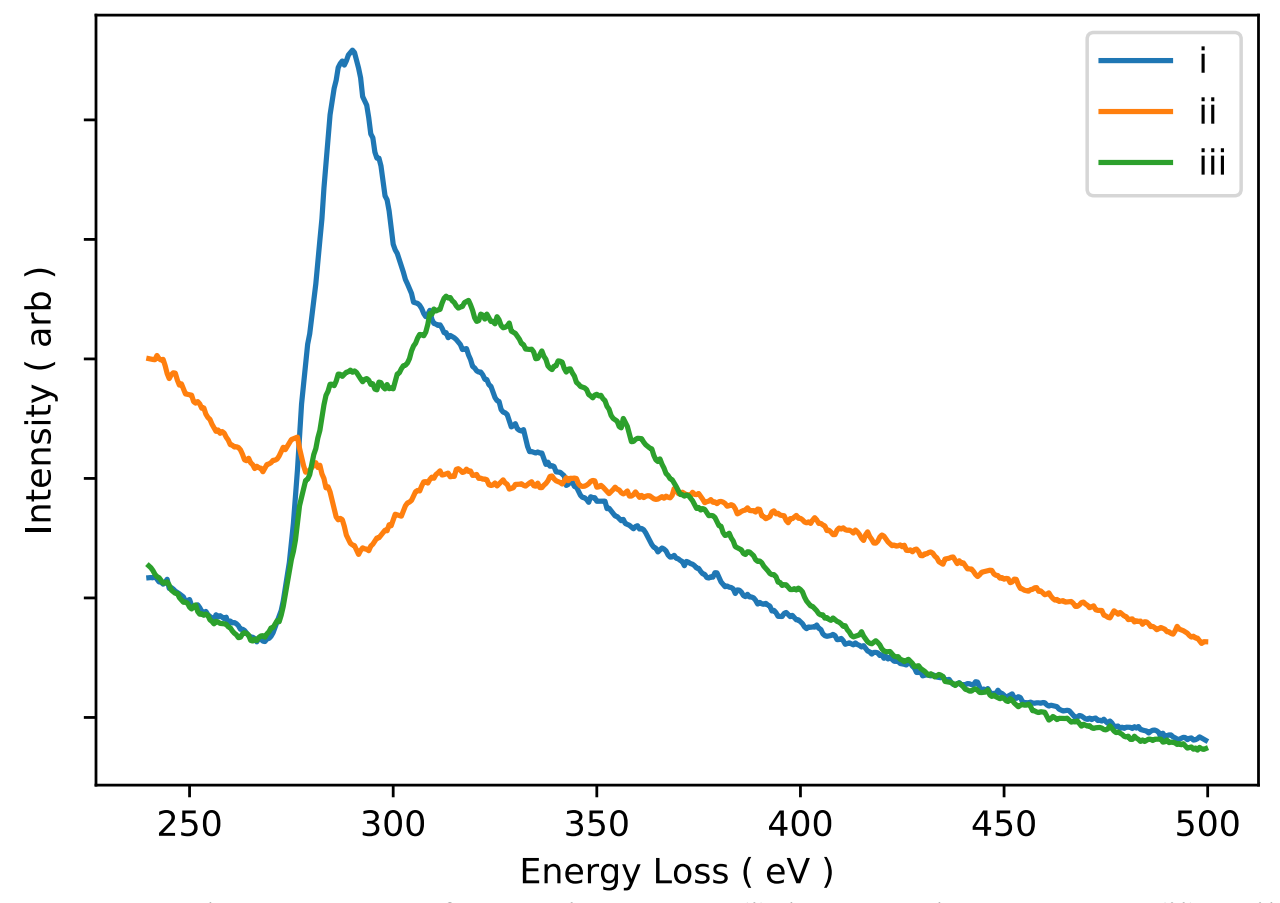

Figure 2. Spectral components from Joint-NMF. (i) lacey carbon support, (ii) palladium component, (iii) carbon contamination. 